Service social

\title{
Des pratiques propices au vivre-ensemble avec des femmes immigrantes au sein d'organisations communautaires
}

\section{Manon Chamberland et Yann Le Bossé}

Volume 60, numéro 1, 2014

Des enfants, des couples et des familles.

URI : https://id.erudit.org/iderudit/1025136ar

DOI : https://doi.org/10.7202/1025136ar

Aller au sommaire du numéro

Éditeur(s)

École de service social de l’Université Laval

ISSN

1708-1734 (numérique)

Découvrir la revue

Citer cet article

Chamberland, M. \& Le Bossé, Y. (2014). Des pratiques propices au vivre-ensemble avec des femmes immigrantes au sein d'organisations communautaires. Service social, 60(1), 100-118.

https://doi.org/10.7202/1025136ar
Résumé de l'article

Les obstacles récurrents rencontrés par des femmes immigrantes, notamment en ce qui concerne leur insertion socioprofessionnelle, ont plusieurs répercussions sur leurs conditions de vie et leur quotidien. Que font les intervenants qui les accompagnent dans leurs démarches ? Cet article présente quatre fondements sur lesquels reposent les pratiques d'intervenantes dans des organisations communautaires de Montréal, Bruxelles et Grenoble. Leurs pratiques rendent compte du développement de solidarités et de savoirs partagés permettant d'exercer un changement sur des obstacles rencontrés quotidiennement par des femmes immigrantes. 


\title{
Des pratiques propices au vivre-ensemble avec des femmes immigrantes au sein d'organisations communautaires
}

\author{
CHAMBERLAND, MANON \\ Professeure \\ Département de travail social \\ Université du Québec en Outaouais \\ LE BOSSÉ, YANN \\ Professeur titulaire \\ Département des fondements et pratiques en éducation \\ Université Laval
}

\begin{abstract}
RÉSUMÉ
Les obstacles récurrents rencontrés par des femmes immigrantes, notamment en ce qui concerne leur insertion socioprofessionnelle, ont plusieurs répercussions sur leurs conditions de vie et leur quotidien. Que font les intervenants qui les accompagnent dans leurs démarches ? Cet article présente quatre fondements sur lesquels reposent les pratiques d'intervenantes dans des organisations communautaires de Montréal, Bruxelles et Grenoble. Leurs pratiques rendent compte du développement de solidarités et de savoirs partagés permettant d'exercer un changement sur des obstacles rencontrés quotidiennement par des femmes immigrantes.
\end{abstract}

Mots-clés : pratiques d'intervention sociale, développement du pouvoir d'agir des personnes et des collectivités, organisations communautaires, femmes immigrantes

\begin{abstract}
The recurring obstacles faced by immigrant women, particularly with regard to their socioprofessional integration, have many impacts on their daily lives. What are the best ways to support them in their efforts? This article presents four foundations upon which the practices of workers in community organizations in Montreal, Brussels and Grenoble are based. These practices reflect the development of solidarity and shared knowledge which help to reduce the obstacles faced daily by immigrant women.
\end{abstract}

Keywords: social practices, empowerment, community organizations, immigrant women 
Depuis les dernières décennies, la plus grande compétitivité entre les entreprises, leur délocalisation et la recherche d'une plus grande flexibilité favorisent le développement d'une économie du savoir (Bernier, 2011) ainsi que plusieurs mutations sur le marché du travail (Paugam, 2004). Ces changements se sont aussi répercutés dans les modalités de soutien aux personnes en transition, ce qui incite à la recherche de pratiques alternatives à des modalités d'accompagnement prescriptives de plus en plus présentes (Gonin, Grenier et Lapierre, 2012 ; Hamzaoui, 2013). Or, en raison de la période de transition que constitue l'immigration, que celleci soit choisie ou non, en raison des défis particuliers auxquels sont confrontées les femmes immigrantes, s'intéresser aux pratiques qui se sont développées pour soutenir leurs initiatives constitue une avenue de recherche pertinente. Dans un premier temps, il sera question des défis auxquels font face les femmes immigrantes pour ensuite exposer dans un deuxième temps, en quoi l'empowerment ou le développement du pouvoir d'agir des personnes et des collectivités constitue une piste prometteuse à explorer pour les pratiques d'intervention sociale avec des femmes immigrantes. Après avoir exposé la méthodologie privilégiée dans le cadre d'une recherche exploratoire et qualitative basée sur l'étude de cas multiples, il sera question des quatre fondements des pratiques des intervenantes au sein d'organisations communautaires fréquentées par des femmes immigrantes de Montréal, Bruxelles et Grenoble. Ces résultats montrent que les intervenantes qui travaillent avec les femmes immigrantes contribuent à agir au quotidien pour développer des solidarités.

\section{DES DÉFIS QUI INCITENT À LA RECHERCHE D'ALTERNATIVES}

À quels défis font face les femmes immigrantes et leur famille ? À ceux que connaissent communément l'ensemble des personnes immigrantes ${ }^{1}$ (langue, développement de nouveaux réseaux, logement, intégration des enfants à l'école, accès au crédit - voir notamment Schellenberg et Maheux, 2007), il semblerait que, pour les femmes, s'ajoute la récurrence de certains obstacles, notamment en ce qui concerne l'insertion et la qualification professionnelle, ainsi que les défis particuliers rattachés à la conciliation famille-emploi et aux études (Normand et Tremblay, 2005). En effet, malgré la diversité de leurs parcours, les difficultés d'une insertion socioprofessionnelle satisfaisante demeurent particulièrement marquées pour les femmes immigrantes, et ce, au sein de plusieurs pays industrialisés. En témoigne un taux de chômage qui, dans certains pays d'Europe, dépasse les $13 \%$ (Belgique et France, par exemple) (OCDE, 2011) et qui, au Canada, est deux fois supérieur à celui des femmes nées au pays. Elles gagneraient aussi des salaires moindres (Conseil du statut de la femme, 2005 ; Fleury, 2007 ; Mongeau, Pinsonneault et Rose, 2007), et ce, même avec une scolarité supérieure à celle des femmes nées au Canada (Beaudoin, 2012 ; Belhassen-Maalaoui, 2008 ; Man, 2004). Leurs conditions liées au fait d'être femme et immigrante compliqueraient parfois sérieusement leurs démarches (Lewis, Gutiérrez et Sakamoto, 2001 ; Man, 2004 ; Pierre, 2005).

En effet, une recherche menée par Chicha (2009), portant sur les trajectoires menant à la déqualification professionnelle chez les femmes immigrantes, fait ressortir que la difficulté, par

\footnotetext{
${ }^{1}$ Bien évidemment, les parcours des femmes immigrantes sont extrêmement diversifiés. L'expression « femmes immigrantes » utilisée dans ce texte ne vise nullement à y référer en tant que groupe homogène. II désigne les femmes qui sont nées à l'extérieur du pays où elles habitent présentement.
} 
exemple, d'avoir accès à des services de garde à différents moments dans leur parcours, ou encore les frais engendrés par les procédures de reconnaissance d'acquis professionnels antérieurs, ainsi que l'accès limité à des réseaux professionnels, contribuent à maintenir les femmes immigrantes dans la précarité. Si l'insertion représente un défi pour les femmes immigrantes qualifiées, qui possèdent déjà les rudiments de la langue parlée dans le pays d'accueil, on peut aisément imaginer que cela s'avère encore plus difficile pour des femmes qui ne maîtrisent pas la langue ou ne possèdent pas de qualification particulière, en cette ère de l'économie du savoir. Cela peut s'avérer encore pire lorsqu'elles sont en attente d'une régularisation de statut. Les pratiques pour soutenir les initiatives des femmes immigrantes nécessitent donc de tenir compte simultanément des obstacles systémiques et personnels que chacune peut rencontrer. Que faire pour soutenir leurs démarches lors de la période de transition que constitue l'immigration?

\section{L'EMPOWERMENT : UNE ALTERNATIVE?}

Dans les dernières décennies, différentes pratiques se sont développées, notamment dans les organisations communautaires pour soutenir les femmes immigrantes et leur famille, ce qui a pu donner lieu à des pratiques novatrices qu'il convient de documenter (Lisboa, 2002 ; VatzLaaroussi, 2008 ; Zentgraf, 2002). En demeurant au plus près des personnes qui les fréquentent, les organisations communautaires se sont historiquement développées à partir de leurs préoccupations. Elles ont tenté de «faire autrement » face à des pratiques qui ne faisaient plus l'unanimité. Ces pratiques alternatives ont été régulièrement associées à l'idée d'empowerment (Duval et al., 2005 ; Wallerstein, 2006). Ce processus désigne le passage entre une situation générant un sentiment d'impuissance réelle ou perçue et l'exercice d'un contrôle réel ou perçu sur cette situation (Gutiérrez, 1990 ; Gutiérrez, 1994 ; Le Bossé, 2004 ; Lee, 2001 ; Rappaport, 1987 ; Tengland, 2008). En tant que processus, ce passage permet d'exercer plus de contrôle simultanément sur les plans personnel et social. II serait rattaché au développement d'habiletés, à l'augmentation du sentiment d'efficacité personnelle, au développement d'une conscience critique et à l'expérience avec d'autres dans une démarche d'action collective (Breton, 1999 ; Breton, 2004 ; Carr, 2003 ; Gutiérrez, 1994).

Or, comment soutenir un tel processus dans le contexte actuel des pratiques d'intervention sociale ? Si l'empowerment favorise la reconnaissance des savoirs détenus par les personnes dans une collectivité donnée, quel peut être l'apport des intervenants sociaux dans cette démarche ? Comme le soulignent Vissandjée et Maillet (2007) : « Malgré les efforts discursifs pour atténuer leur rôle, ils ressurgissent constamment et, davantage encore, demeurent des acteurs centraux » (p. 145). Cette citation souligne l'importance de s'interroger sur les pratiques d'intervention pertinentes pour soutenir l'empowerment. Les développements théoriques menés à propos de l'empowerment ont permis d'identifier plus précisément une série de principes à mettre en œuvre dans le cadre des pratiques d'intervention sociale.

Plus spécifiquement, la modélisation proposée par Lee (2001) en travail social a permis de tenir compte des situations auxquelles font face les femmes immigrantes à travers un prisme qui prend en considération les multiples déterminants et discriminations qu'elles peuvent rencontrer mais aussi les stratégies qu'elles déploient (vision historique, perspective ethnique et de classe, 
perspective multiculturelle, perspective féministe, perspective globale, perspective critique). La modélisation proposée par Lee (2001) rejoint en cela une approche intersectionnelle qui considère les croisements des discriminations potentielles, notamment dans les rapports sociaux et qui influencent la manière dont les personnes se perçoivent tout comme les occasions présentes dans leur environnement, ce qui joue dans les actions qu'elles posent. En counseling, McWhirter (1994; 1998) a élaboré une modélisation, les « 5 C» (conscience critique, contexte, compétence, collaboration, communauté) qui rend compte de cinq principes d'intervention sociale, et ce, sur les plans interpersonnel, organisationnel et communautaire.

De plus, du côté francophone, les travaux menés plus spécifiquement par Le Bossé (2004; 2010 ; 2012) ont permis de préciser conceptuellement le terme empowerment et d'en proposer une traduction à partir d'une revue de littérature exhaustive sur le sujet. En effet, l'expression « développement du pouvoir d'agir des personnes et des collectivités » (DPA) semble plus à même de rendre compte de ce processus. Les travaux de Le Bossé ont aussi permis de dégager quatre composantes d'un cadre d'analyse des pratiques, qui souligne l'importance de tenir compte prioritairement des personnes directement concernées par la poursuite d'un changement, mais aussi de l'ensemble des acteurs impliqués dont les enjeux peuvent interférer avec la démarche. Ces quatre composantes sont : 1) l'adoption de l'unité d'analyse acteur en contexte ; 2) la prise en compte des contextes d'application ; 3) la définition du changement visé et de ses modalités avec les personnes concernées ; 4) l'introduction d'une démarche d'action conscientisante (Le Bossé, 2010; Le Bossé, 2012). De ces travaux, nous retenons ici l'expression « développement du pouvoir d'agir des personnes et des collectivités »(DPA) ${ }^{2}$ pour désigner le processus d'empowerment. Bien que cela soit implicite dans plusieurs écrits sur le DPA (Breton, 1999 ; Breton, 2004 ; Gutiérrez, 1990 ; Le Bossé, 2004 ; Lee, 2001 ; McWhirter, 1998 ; Ninacs, 2008), ce processus implique le développement et la redécouverte de savoirs issus du passage à l'action.

Compte tenu que le DPA est un processus qui se développe dans l'intersubjectivité, cela conduit à s'intéresser à la manière dont les intervenants conçoivent leur rôle et travaillent avec les femmes immigrantes pour demeurer au plus près de la définition de ce qu'elles souhaitent changer. Cela implique aussi de s'attarder aux fondements de leurs pratiques. D'une part, s'intéresser de plus près aux pratiques des organisations communautaires qui soutiennent l'intégration sociale et professionnelle ${ }^{3}$ des femmes immigrantes qui les fréquentent permet de documenter de quelle manière les intervenants ont développé des pratiques novatrices au diapason de leurs réalités. D'autre part, étant donné les multiples changements survenus dans les dernières années dans ce type d'organisation (professionnalisation, développement de partenariats avec l'État, financement par projets, etc.) (Le Goff et al., 2005), le fait de s'intéresser à ces pratiques permet de s'interroger sur la façon dont les intervenants poursuivent ce travail d'innovation et contribuent au DPA malgré tout et en prenant appui sur ces

\footnotetext{
${ }^{2}$ La démarche empruntée ici se distingue des développements conceptuels de Le Bossé dont les travaux ont donné lieu à une approche particulière des pratiques. Voir à ce sujet Le Bossé, Y. (2010). Psychosociologie des sciences de l'orientation : un point de vue interactionniste et stratégique. Québec : Éditons ARDIS.

${ }^{3}$ L'intégration réfère ici au sens où l'entend Schnapper (2007) : « Qu'on évoque la régulation, l'intégration, le "faire société", le "vivre ensemble" ou le "lien social", l'interrogation reste la même, elle porte à la fois sur l'intégration des individus à la société et sur l'intégration de la société dans son ensemble. » (p. 16)
} 
transformations. Que font les intervenantes avec les femmes immigrantes dans les organisations communautaires ${ }^{4}$ pour soutenir le DPA ? Quelles composantes des pratiques des intervenantes avec des femmes immigrantes au sein d'organisations communautaires, destinées à soutenir l'intégration sociale et professionnelle, semblent propices au développement du pouvoir d'agir des personnes et des collectivités ? Quels sont les savoirs mobilisés par les intervenantes dans leur pratique?

\section{MÉTHODOLOGIE}

Une recherche qualitative et exploratoire, basée sur l'étude de cas multiples (Roy, 2003), a permis de cerner des pratiques mises en œuvre au sein de cinq organisations communautaires fréquentées par des femmes immigrantes ${ }^{5}$.

\section{Sélection des sites à l'étude}

Une liste de 67 organisations communautaires situées à Montréal et fréquentées par des femmes immigrantes, établie à partir des répertoires de ressources, fut réduite à 25 organisations, hiérarchisées selon une grille d'analyse construite suivant l'idéal-type des organisations communautaires identifié par René (2005), la grille d'analyse du DPA, fondée sur le modèle des « $5 \mathrm{C}$ » de McWhirter (1998), ainsi que les indicateurs de participation identifiés par Rifkin (2003) en santé communautaire ${ }^{6}$. Cinq appels téléphoniques auprès des cinq premières organisations communautaires de la liste ont permis d'obtenir la participation de deux organisations. La première, fondée par un groupe de femmes d'un même quartier qui souhaitaient avoir un lieu pour se rencontrer et réaliser des activités, a pignon sur rue depuis un peu plus de quinze ans. Elle s'adresse à des femmes de toutes origines et leur offre l'occasion de participer à des comités et à des activités ponctuelles ou, tout simplement, de venir échanger et rencontrer d'autres femmes (Org. A). La deuxième organisation est un projet-pilote, mis en place au cours des cinq dernières années qui offre une formation de dix mois à des femmes immigrantes où elles ont l'occasion de se familiariser avec les ressources du quartier et de la ville et de développer une pratique d'accompagnement des familles nouvellement arrivées dans le quartier et qui rencontrent des obstacles ponctuels dans leur démarche d'installation. (Org.B).

Afin de diversifier les contextes à l'étude, furent ajoutées deux organisations communautaires de Bruxelles en Belgique et une de Grenoble en France, référées par des informateurs-clés, et

\footnotetext{
${ }^{4}$ Le terme « organisation communautaire » est préféré à celui d'« organismes communautaires » dans cette recherche afin de désigner ces espaces, ancrés dans la communauté, le plus souvent issus de personnes qui se sont regroupés sur la base de préoccupations communes et qui ne font pas nécessairement partie du mouvement de l'action communautaire autonome.

${ }^{5}$ Chamberland, M. (2014). Le développement du pouvoir d'agir des personnes et des collectivités : un aperçu des pratiques avec des femmes immigrantes au sein d'organisations communautaires de Montréal, de Bruxelles et de Grenoble. Thèse de doctorat. Québec : Université Laval.

${ }^{6}$ Les six critères utilisés dans cette étude pour sélectionner les organisations communautaires sont : 1) la possibilité de s'impliquer dans des activités propices à utiliser ses forces et ses compétences ; 2) être désignée comme une personne digne et ayant des droits ; 3 ) la possibilité de pouvoir exercer un changement dans sa situation personnelle à partir de sa propre définition du changement poursuivi et de ses retombées ; 4) la possibilité de pouvoir participer d'une manière ou d'une autre aux instances décisionnelles ; 5) la possibilité de s'impliquer de différents manières et de connaître d'autres personnes; 6) la possibilité de pouvoir participer à des activités qui rassemblent tant des personnes immigrantes que la population (communauté géographique et / ou d'intérêt).
} 
en conformité avec les critères de la grille d'analyse. À Bruxelles, les deux associations sans but lucratif retenues concentrent leurs activités en alphabétisation et en éducation permanente. Mises sur pied pendant les années 1970, la première s'adresse exclusivement aux femmes d'origines étrangères (Org. C) et se situe dans un quartier à forte densité de personnes immigrantes. La deuxième, fréquentée par plusieurs femmes d'origines diverses, arrivées récemment en Belgique ou depuis plusieurs années, est mixte et s'adresse tant aux natifs belges qu'aux personnes d'origine étrangère. Cependant, celle-ci est majoritairement fréquentée par des femmes qui viennent de partout dans le monde (Org. D). À Grenoble, l'association sans but lucratif participante, mise sur pied en 2009, se veut un lieu d'échange entre les mamans seules et leurs enfants. Sa mise sur pied fait suite à une série de réflexions autour de la précarité et de l'éducation des enfants (Org. E).

\section{Méthodes de collecte et d'analyse de données}

Au total, 13 intervenantes ${ }^{7}$ ont pris part à une à deux entrevues semi-dirigées individuelles d'une durée de cinquante minutes à une heure trente, après avoir pris connaissance du formulaire de consentement exposant les objectifs de la recherche, la participation attendue et ses implications ainsi que des précautions concernant la confidentialité. Le guide d'entretien portait sur : 1) les stratégies d'intervention privilégiées par l'organisme et par les intervenantes et la manière dont elles se déroulent ; 2) la conception des intervenantes de l'accompagnement des femmes immigrantes ; 3) les obstacles rencontrés dans le cadre de leur pratique quotidienne et les éléments facilitants perçus ; 4) la description d'un incident critique ${ }^{8}$ rattaché à leur pratique. Également, dans chaque organisation, une entrevue en groupe, d'une durée d'une heure trente à trois heures, a permis de rencontrer de trois à huit femmes immigrantes. L'ensemble des entrevues ont été enregistrées et retranscrites mot à mot. En plus de recourir à l'étude de cas, cette recherche s'inspire du principe de l'émergence des données de la méthodologie de la théorisation enracinée, envisagée plutôt ici comme un projet épistémologique (Luckerhoff et Guillemette, 2012). L'analyse inductive du contenu manifeste des entrevues a permis de dégager des thèmes émergents à la suite de plusieurs lectures et de différentes étapes de codage (Charmaz, 2006). Les entrevues réalisées avec les intervenantes ont été analysées en fonction de chaque cas pour ensuite faire l'objet d'une analyse transversale (l'ensemble des cas) (Creswell, 2013), ce qui a permis d'arriver aux résultats suivants.

\section{RÉSULTATS}

Quelles pratiques des intervenantes avec des femmes immigrantes, au sein d'organisations communautaires destinées à soutenir leur intégration sociale et professionnelle, semblent les plus propices au développement du pouvoir d'agir des personnes et des collectivités ? L'analyse

\footnotetext{
${ }^{7}$ À noter que pour des raisons de concision, dans les sections « résultats » et « discussion », l'ensemble de ces quatorze personnes est désigné par le vocable «intervenantes » plutôt que de distinguer systématiquement les intervenantes des coordonnatrices.

${ }^{8}$ Un incident critique est un événement survenu qui provoque une rupture dans les manières habituelles de faire et de penser. Pouvant paraître anodin au premier regard, il s'avère pourtant particulièrement propice aux apprentissages, car il permet de réfléchir à ses propres cadres de références et à ses présupposés (CohenÉmérique, 1993 ; Leclerc, Bourassa et Filteau, 2010 ; Legault et Roy, 2000).
} 
transversale des données recueillies montre que les actions des intervenantes reposent sur quatre fondements interreliés d'une intervention propice au DPA, à savoir :

- l'expérience comme fondement de la connaissance ;

- le quotidien comme contexte d'application ;

- une pratique intégrée au quartier ;

- une pratique porteuse de sens.

\section{L'expérience comme fondement de la connaissance}

L'expérience comme fondement de la connaissance constitue la raison d'être des organisations communautaires. L'expérience comme fondement de la connaissance permet aux femmes immigrantes tout comme aux intervenantes qui les accompagnent de se rassembler, de s'interroger, de se mettre à l'épreuve et de se projeter.

\section{Pour se rassembler}

Il est rapidement ressorti des entrevues réalisées avec les intervenantes de l'ensemble des organisations communautaires participantes que leur fondation reposait sur la volonté des femmes de créer un lieu pour échanger. Ainsi, ce n'est pas sur la base des problèmes rencontrés par les femmes immigrantes que sont définies les missions et les activités des organisations communautaires, mais bien sur une formulation qui rend compte du potentiel des femmes et de leur désir d'apprendre. L'ensemble des organisations privilégie aussi le travail en groupe. À partir de ce rassemblement, l'expérience devient une base pour s'interroger.

\section{Pour s'interroger}

En fait, il est intéressant de constater que les intervenantes se positionnent elles-mêmes en tant qu'apprenantes. Elles vont chercher à comprendre comment chaque femme définit sa situation.

C'est en parlant aussi qu'elle va clarifier d'où le besoin de l'écoute, c'est ça que c'est important. L'échange, [...] il est fait dans une clarification réciproque parce que si tu me racontes ce qui s'est passé, c'est pour mieux comprendre mais avec toi [...] Donc, c'est ça valider les sentiments, aller voir ensemble les informations et puis c'est elle qui va trouver les solutions. (Maria, Montréal, Org. A)

Autrement dit, les intervenantes n'adoptent pas un rôle d'experte qui sait et qui « doit éveiller les consciences ». Elles s'efforcent plutôt de mettre en place les conditions d'échanges entre les femmes pour qu'elles partagent leurs expériences et deviennent ainsi une source de savoir.

C'est-à-dire que, effectivement, on n'est pas tout seul, et donc du coup, si on peut expérimenter de temps en temps, on peut échanger avec d'autres sur des questions d'éducation, même si ça dure dix minutes, je dis ça aussi parce que je suis vraiment convaincue de l'importance de l'expérience. (Judith, Grenoble, Org E)

La redécouverte ou le développement de savoirs s'effectue souvent en groupe dans une forme de mise à l'épreuve où il y a des défis à relever. 
Pour se mettre à l'épreuve avec d'autres

L'organisation devient un lieu où il est possible, par exemple, de soulever certaines questions.

Ben, je pense que ça, ça fait partie du projet, c'est-à-dire offrir un espace à des personnes où elles peuvent expérimenter un certain nombre de choses, ou dire ce qu'elles ont à dire, et qu'elles ne seront pas jugées dans les paroles qu'elles diront. (Caroline, Grenoble, Org E)

Cette participation est une forme de mise à l'épreuve avec d'autres femmes ce qui a des retombées bien au-delà de l'expérience de chacune :

Donc suite à ça [une pièce de théâtre réalisée par des femmes immigrantes], ça a donné à d'autres l'envie de vivre d'autres expériences et on a mis d'autres ateliers en place qui sont devenus hebdomadaires, et qu'il y a d'autres femmes qui ont aussi participé et il y a un deuxième spectacle qui a été créé. (Sophie, Bruxelles, Org. C)

Cette mise à l'épreuve, qui consiste à relever des défis ou à accomplir une action comportant une dimension publique, ne se réalise pas seulement avec les autres femmes. En effet, que ce soit dans le cadre de leurs interventions ponctuelles, d'ateliers hebdomadaires ou de projets qui rassemblent plusieurs personnes de la collectivité ou de différents milieux, les intervenantes s'assurent que chaque occasion de rencontres permet de mettre en valeur l'expérience des femmes immigrantes. Ce n'est donc pas uniquement le résultat qui compte mais également le processus qui y conduit. Les intervenantes font le postulat qu'accomplir une action avec d'autres et dont les retombées se remarquent au-delà de l'organisation se révèle être une expérience pouvant être transformatrice.

\section{Pour se projeter}

L'expérience constitue une source d'apprentissages qui permet non seulement aux femmes de relire leur propre histoire et de la partager, mais aussi de se projeter.

Ce sont des femmes qui entament une démarche de réflexion sur elle-même. $Y$ en a une qui va retourner aux études, parce qu'elle a beaucoup aimé cette expérience-là, et l'autre aussi va changer de travail. (Maria, Montréal, Org. A)

Ces expériences sont issues du quotidien des femmes, ce qui constitue un deuxième fondement majeur des pratiques au sein des organisations communautaires participantes.

\section{Le quotidien comme contexte d'application}

Dans l'ensemble des organisations étudiées, l'espace que constituent ces organisations communautaires est défini comme un milieu de vie où l'informel est privilégié. En témoigne l'organisation des locaux où l'on retrouve des salles propices aux échanges et au travail en groupe plutôt que des bureaux fermés où l'on est accueillie sur rendez-vous. Ces organisations communautaires deviennent ainsi des espaces non stigmatisants, où il est possible de sortir de la solitude. C'est aussi en se basant sur le quotidien que les intervenantes font ressortir les compétences que les femmes détiennent. 
[...] par exemple, l'atelier théâtre qu'on a mis en place, y a trois ans, y a quelques femmes qui ont créé un spectacle à partir de récits sur leur arrivée en Belgique et qu'après elles ont présenté sur scène plusieurs fois, et donc là, on voit donc le trajet qu'elles ont fait. Elles ne se seraient jamais imaginé monter sur scène et s'emparer de cet outil d'expression pour aussi sensibiliser d'autres personnes sur leurs parcours, ce qu'elles ont vécu, ce qu'elles sont. (Sophie, Bruxelles, Org. C)

Les intervenantes misent prioritairement sur ce point d'appui que constitue le quotidien. À partir de cela, elles dégagent de nouveaux projets pour faciliter l'accès des femmes aux ressources et mettre en place les conditions propices à initier des actions qui contribuent à transformer les obstacles rencontrés en occasions d'apprentissages. Les initiatives mises en place par les femmes dans ces organisations ont des retombées concrètes dans le quartier, ce qui constitue un troisième fondement.

\section{Des pratiques intégrées au quartier}

L'ensemble des organisations présente la particularité d'être bien intégrées dans le quartier où elles se situent. On y vient par le bouche-à-oreille. Les intervenantes développent aussi différentes collaborations avec d'autres organismes et instances du quartier. Plusieurs autres organisations y sont aussi présentes dont certaines ont été créées à l'initiative de femmes immigrantes qui ont fréquenté les organisations retenues dans cette recherche. C'est le cas de l'organisation C. À travers les différentes vagues d'immigration, les initiatives mises en place dans ces organisations communautaires ont fait en sorte de rendre les réalités des femmes immigrantes et de leur famille bien visibles aux yeux des décideurs, mais par le truchement des réalisations des femmes. Ces initiatives, bien ancrées dans le quotidien des femmes immigrantes, de leurs familles et de la collectivité dans laquelle elles s'inscrivent, contribuent à tisser un lien entre les personnes du quartier.

Il faut qu'il y ait des réseaux aussi qui se créent puis on se rend compte que si une famille est venue ici, souvent, elle en parle à la voisine. Tu sais, le bouche à oreilles c'est incroyable. Puis en même temps c'est sûr que des fois, la personne qui a reçu un service ici, elle se souvient de ce qu'on lui a dit ou comment ça s'est passé, puis elle donne l'information directement à sa voisine. Donc, la voisine n'a pas nécessairement besoin de venir ici. Donc c'est intéressant. (Julie, Montréal, Org. B)

II en résulte un sentiment d'appartenance manifeste dans les propos des femmes immigrantes qui fréquentent ces organisations communautaires et la conviction de la part des intervenantes qu'il y a bien une solidarité qui s'établit dans le quartier. Alors que, dans les médias en général, lorsqu'on traite d'immigration, on entend le plus souvent parler de conflits, rattachés à la transmission de la culture ou à l'importance de sauvegarder le français, qui placent ces questions dans des rapports d'opposition (population d'accueil - personnes immigrantes), les propos des intervenantes laissent entrevoir des réalités tout à fait différentes et rappellent que cette transmission et ces transformations s'effectuent au quotidien.

Je pense que leur apport, il faut le voir dans la vie de tous les jours, au quotidien dans l'échange qu'elles ont. Quand elles échangent sur leur expérience, cela crée une ouverture. C'est ça l'apport et ce sont ces petites choses-là qui font qu'[à] un moment donné, quand on va avoir 
besoin de solidarité, elles vont être là. Parce qu'on leur aura permis de connaître les autres femmes. (Suzanne, Montréal, Org. A)

II en résulte la conviction qu'il y a toujours lieu de créer des liens de solidarité, ce sur quoi repose le sens que les intervenantes retrouvent même après plusieurs années dans cette organisation communautaire.

\section{Des pratiques porteuses de sens}

Cette question du sens derrière les actions posées ressort clairement des entretiens réalisés avec les intervenantes et constitue un quatrième fondement. Tant pour celles qui sont à l'emploi de ces organisations communautaires depuis peu que pour celles qui y sont depuis quinze, vingt, voire trente ans, le sens qu'elles donnent à leur travail explique en majeure partie pourquoi elles y sont toujours. Mais sur quoi repose plus spécifiquement ce sens ?

II ressort des propos des intervenantes concernant le sens de leur travail que chaque journée apporte son lot de défis et qu'elles sont ainsi en position d'apprendre constamment dans leur pratique. "Parce qu'ici, c'est un boulot où tu apprends tout le temps, tout le temps. Tu pourras jamais dire : ben moi, dans dix ans, j'ai fini ma carrière. » (Valérie, Bruxelles, Org. D) Travailler avec des femmes immigrantes est un enrichissement pour l'ensemble des intervenantes rencontrées. II s'agit d'un travail qui demande de s'adapter constamment.

Alors je pense que le mot que je mettrais, c'est l'adaptation. II faut t'adapter. Tu t'adaptes à la vague qui est là. Tu t'adaptes aux femmes qui sont là. Et je pense que pour moi, de me retrouver dans un centre qui serait monoculturel, je m'en irais là, parce que la richesse, c'est ça. (Suzanne, Montréal, Org. A)

Ce qui caractérise plus particulièrement les organisations participantes et qui se retrouve au cœur des pratiques des intervenantes est la question de l'accueil, sur lequel repose en grande partie le sens de leur travail et qui devient vecteur de solidarités à partir des points communs. " Alors plutôt que de regarder les différences, commençons par les ressemblances. [...] C'est comme s'il y avait dix crochets à attacher, et puis si y en a déjà quatre d'attachés avec nos ressemblances, ben mon Dieu, on a ça en commun et le reste on peut peut-être commencer à en parler. » (Suzanne, Montréal, Org. A)

Les initiatives mises en place avec les femmes ainsi que leur manière de porter un regard neuf sur chaque situation qu'elles rencontrent démontrent une créativité de pensée et d'action. Par la connaissance fine qu'elles développent au contact quotidien des femmes immigrantes, les intervenantes constatent non seulement les obstacles auxquels elles font face mais aussi leurs actions pour les surmonter. Ce faisant, elles développent la conviction qu'elles peuvent agir au quotidien pour faire reconnaître l'expertise des femmes immigrantes auprès d'autres acteurs.

\section{Agir avec les femmes immigrantes: rassembler et mobiliser}

En résumé, l'ensemble de ces quatre fondements interreliés fait en sorte que les intervenantes rencontrées dans le cadre de cette recherche agissent au quotidien pour soutenir les initiatives 
des femmes immigrantes, par leur engagement envers leur travail et envers elles, ainsi qu'envers la vision qu'elles mettent en œuvre. Que ce soit dans le fait de prendre le temps de traduire au moins en anglais et en français l'ensemble des documents pour faciliter l'accès à l'information et soutenir la participation (Org. A), de s'ajuster au rythme de chacune, d'être attentives à remettre en question ce qu'elles pourraient prendre pour acquis, les intervenantes agissent résolument en tant que vigiles. En témoignent les projets initiés, comme, par exemple, un service de garde mis en place suite au constat que les femmes immigrantes en formation se butaient à l'obstacle récurrent de ne pas avoir accès à des places en garderie.

Donc je dirais même plus que ça, parce qu'on l'a créé [ce service de garde] et, en même temps, on voulait que ce soit les femmes qui n'avaient pas de formation et qui étaient en Alpha qui prennent ça en charge parce qu'elles avaient exprimé que c'était leur projet professionnel. (Béatrice, Bruxelles, Org. C)

C'est en ce sens qu'elles n'hésiteront pas à interpeller d'autres acteurs en plaçant au centre de leurs préoccupations l'expérience concrète des femmes et leur expertise expérientielle.

Oui, on le fait maintenant avec toute l'équipe et les parents [de l'accueil d'enfants], on a fait dans les années 2008-2009 une vidéo qui montre toute la problématique des mères en formation, en recherche d'emploi, seules avec enfants et qui ne trouvent pas de crèche. Et on a interpellé le ministre, hein !, qui est venu et donc on a, c'est aussi avec le public qu'on fait cette interpellation, avec la vidéo qu'on a réalisée dans ce cadre et donc qui a très, très bien fait. C'est aussi, on essaie, tu vois, de sensibiliser avec le public, donc de donner la parole à elles de défendre ce qui est vraiment important pour elles, donc on garde cet aspect très fort [...]. (Béatrice, Bruxelles, Org. C)

Ces pratiques démontrent qu'ensemble, les intervenantes et les femmes immigrantes réussissent à établir des ponts au quotidien qui constituent autant de points d'appui pour réfléchir à la question de l'action dans les organisations communautaires. Leurs expériences incitent à remettre en question ce qui peut apparaître comme une évidence : qu'agir ensemble est de moins en moins possible. Parce qu'elles savent comment voir ce qui est important pour les femmes immigrantes, les intervenantes mettent en place les conditions propices à des projets donnant lieu à la mise en récit et à la relecture de leur expérience devenue publique. Dans ces organisations communautaires, là où il y avait comme une forme de repli, une perte de sens et de créativité pour agir, se tissent chaque jour des liens vecteurs de solidarité.

\section{DISCUSSION}

\section{L'expérience comme fondement de la connaissance}

En prenant appui sur l'expérience que détiennent les femmes immigrantes, les intervenantes privilégient concrètement les rapports où un dialogue peut s'instaurer au sens où l'entend Freire (1998). Elles contribuent donc à établir des rapports qui se veulent le plus possible égalitaires, des rapports de personnes humaines égales en droit qui, devant une situation en apparence insoluble, se placent en position de «co-réflexion ». Comme le soulignent plusieurs auteurs, les personnes accompagnées détiennent des savoirs et une expertise que les intervenants n'ont pas nécessairement et qui sont complémentaires à l'expertise professionnelle de ces derniers 
(Lee, 2001 ; Le Bossé, 2012 ; Tengland, 2012). Judith A. Lee (2001) estime que les intervenants sociaux doivent s'intéresser aux réalités des personnes susceptibles de faire face à des discriminations en tenant compte de leur expérience et de leur point de vue. Elle invite les intervenants à écouter les histoires de vie des personnes qu'ils rencontrent en tenant compte d'une grille d'analyse à sept dimensions. Dans son modèle basé sur les « $5 \mathrm{C}$ », McWhirter (1998) propose aux intervenants d'établir des rapports de collaboration et de tenir compte des compétences détenues par les personnes. De même, Le Bossé (2010) propose dans sa grille d'analyse à quatre axes de définir le problème et les solutions en recourant aux expertises combinées des intervenants et des personnes accompagnées. Rifkin (2003) et ses collègues (Rifkin et al., 1988), qui se sont surtout attardés à opérationnaliser le concept de participation qui est rattaché étroitement au DPA, soutiennent que les organisations où les personnes ont le plus de chances de développer leur DPA sont celles qui reconnaissent les habiletés et les savoirs des personnes qui les fréquentent et qui leur donnent l'occasion d'en développer d'autres.

Or, que font justement les intervenantes rencontrées ? Elles mettent en place des possibilités de participation formelles et informelles pour les femmes immigrantes qu'elles rencontrent et selon ce qu'elles souhaitent faire. Cette place accordée à l'informel serait particulièrement propice à développer des liens de réciprocité et de reconnaissance (René, 2009), vecteurs d'actions collectives. Cette richesse de l'informel prend place dans le quotidien.

\section{Le quotidien comme contexte d'application}

Par les connaissances fines qu'elles développent de ce que vivent les femmes immigrantes, les intervenantes sont à même de considérer leur situation de manière complexe. Ce sont ici les femmes immigrantes qui sont expertes de leur quotidien. En cela, ce fondement rejoint la modélisation conceptuelle proposée par Lee (2001) où les intervenants sont invités à considérer le contexte dans lequel se retrouvent les personnes qu'ils rencontrent où celles-ci rendent compte, à travers leurs histoires, des dimensions qui peuvent être source de discriminations. Or, ces discriminations prennent forme dans le quotidien. Cela rejoint également la composante « contexte » de la modélisation conceptuelle de McWhirter (1998) tout comme celle de Le Bossé (2010) qui invite à tenir compte de ce qui est important « ici-et-maintenant ». En ce qui concerne les propositions de Rifkin (2003), il semble bien que la circulation et l'accès des informations au quotidien aient plus de chances de donner lieu à une vision partagée des développements de l'organisation, de représenter l'ensemble des intérêts des personnes concernées, de favoriser les accords à propos des rôles et des responsabilités ainsi que des moyens pour résoudre les conflits qui peuvent survenir. De plus, dans chacune des organisations, il y a plusieurs possibilités de participation à des comités où s'impliquer et prendre part aux processus décisionnels. Mais surtout, à chaque instant, les pratiques des intervenantes démontrent qu'elles ont le souci de dégager des possibilités de choix afin que les femmes puissent exercer leur décision, ce qui ressort de l'ensemble des incidents critiques.

C'est aussi dans ce souci de prendre appui sur le quotidien qu'elles parviennent à faire ressortir les situations individuelles qui, mises en commun, deviennent un enjeu collectif. Celui-ci une fois identifié, il permet d'entreprendre avec d'autres acteurs des initiatives qui ont des 
retombées concrètes dans le quartier. En témoigne l'exemple de la mise en place du service de garde pour les femmes immigrantes en formation qui n'y avaient pas accès dans l'organisation C.

\section{Des pratiques intégrées au quartier}

Les pratiques des intervenantes dans les organisations participantes ont des retombées concrètes dans le quartier qui contribuent à favoriser la création de liens et de réseaux, non seulement entre les femmes, mais aussi avec d'autres acteurs de la collectivité. Ces réseaux contribuent à faciliter l'établissement en pays d'accueil (Deville-Stoetzel et al., 2012). Les initiatives mises en place par les femmes immigrantes contribuent à faire partager leur expérience, à faire connaître leurs réalités et à interpeller différents acteurs à l'égard des obstacles qu'elles rencontrent et de ce qu'elles peuvent apporter à la collectivité. Par exemple, dans l'organisation B de Montréal, les accompagnements effectués par les femmes immigrantes contribuent à créer des liens entre des acteurs qui autrement ne se seraient pas parlé (parents, employés des commissions scolaires, responsables d'organismes communautaires, municipaux et gouvernementaux, etc.). Il en est de même dans l'organisation $C$ de Bruxelles où la mise sur pied du service de garde a pu sensibiliser les décideurs. Ces exemples démontrent que les organisations sont bien implantées dans le quartier et deviennent des structures médiatrices dans lesquelles les femmes immigrantes peuvent s'impliquer et poser des actions qui ont des impacts dans la collectivité. Cela rejoint la modélisation à sept dimensions de Lee (2001) où ces initiatives ont des retombées simultanées dans la manière dont les femmes immigrantes perçoivent leur situation et dans les possibilités ainsi créées dans la collectivité. II en est de même pour la modélisation de McWhirter (1998) et la composante « communauté » qui invite à favoriser les relations avec la collectivité ainsi qu'à soutenir l'implication sociale des personnes. En ce qui concerne la modélisation conceptuelle à quatre axes de Le Bossé (2010), elle invite à considérer les enjeux de différents acteurs dans la définition du problème par la composante «acteurs-en-contexte ». Cet auteur encourage aussi les intervenants à ne pas limiter leur accompagnement à la personne mais aussi de faire ressortir les possibilités d'actions sur le plan collectif. En ce qui concerne le modèle de Rifkin (2003), qui vise entre autres à améliorer l'environnement, cela implique notamment de tenir compte des liens établis dans le quartier et du sentiment d'appartenance que les personnes peuvent y développer. Ce sont ces retombées qui contribuent, entre autres, à ce que les intervenantes trouvent toujours du sens à leur travail.

\section{Des pratiques porteuses de sens}

Les résultats de cette recherche démontrent que les intervenantes entretiennent le sens de leur travail même après plusieurs années dans des conditions qui ne s'avèrent pas toujours faciles. Ce sens est rattaché aux liens qu'elles contribuent à créer et à entretenir avec les femmes immigrantes mais aussi aux retombées de leurs actions pour le changement social. II est à remarquer que le fait de pouvoir partager en équipe des questionnements quant à leur pratique, d'avoir des espaces d'échange propices aux mises en commun pour apprendre avec les autres, à partir de l'évaluation des activités et des projets, par exemple, contribuent à enrichir le quotidien du travail des intervenantes. La plupart des intervenantes rencontrées accordent aussi une importance à la liberté que leur permet leur travail. En effet, leur travail n'est pas routinier, 
leur quotidien est ponctué d'imprévus : chaque occasion de rencontre peut donner lieu à un projet défini à partir de ce qui se révèle important pour les femmes. Ce faisant, leur travail les place constamment devant la nécessité de co-créer des possibilités de participation significative avec les femmes, et ce, à partir de leurs compétences et de leurs savoirs. Plutôt qu'un cadre où ce seraient les femmes immigrantes qui auraient à s'ajuster aux organisations et à ce qui s'y fait, c'est plutôt une logique d'émergence qui est privilégiée, où il est permis d'imaginer un autre monde possible, et ce, même au sein des organisations plus grandes ( $C$ et $D$ ). En ce qui concerne les modélisations conceptuelles de Lee (2001) et de McWhirter (1998), cela peut se rattacher à la composante de la conscience critique. Dans la modélisation conceptuelle de Le Bossé (2010), cela pourrait se rapprocher de l'introduction d'une démarche d'action conscientisante où un retour systématique est effectué afin de dégager les apprentissages, évaluer les retombées des actions posées de manière à envisager la suite. Dans le modèle de Rifkin (2003), tant les salariés d'une organisation que la population à laquelle elle s'adresse devraient pouvoir s'impliquer concrètement dans les décisions et dans les orientations qui la concernent. Or, les cinq organisations participantes ont toutes, à des degrés divers, des mécanismes permettant la libre circulation de l'information et des occasions de participation volontaires aux orientations.

En ce qui concerne plus spécifiquement le processus du développement du pouvoir d'agir des personnes et des collectivités, Rifkin (2003) pose ouvertement la question suivante : le développement du pouvoir d'agir des personnes et des collectivités peut-il reposer sur les intervenants qui les accompagnent ou ces personnes et collectivités ne doivent-elles pas développer ce pouvoir d'agir elles-mêmes ? Posée ainsi, la question semble insoluble. Les résultats présentés dans cet article laissent plutôt croire que le développement du pouvoir d'agir des personnes et des collectivités est un processus qui se construit dans un aller-retour incessant entre les intervenantes et les femmes immigrantes. En effet, comme l'expérience est au fondement de la connaissance, en s'attardant non seulement aux obstacles rencontrés par les femmes mais aussi aux stratégies qu'elles déploient pour s'en sortir, en misant sur le quotidien comme contexte d'application, il semblerait que les intervenantes font continuellement preuve de créativité avec les femmes et d'autres acteurs de la collectivité. Ce faisant, la satisfaction que les intervenantes éprouvent à constater que les femmes immigrantes développent leur pouvoir d'agir et qu'elles contribuent concrètement à créer des collectivités plus inclusives, le fait qu'elles ont toujours l'impression d'apprendre serait rattaché à leur propre pouvoir d'agir. II en résulte aussi des occasions de rencontres qui sont propices au développement de connaissances partagées pour soutenir le changement social. S'intéresser de plus près aux pratiques des organisations communautaires rend compte qu'elles sont toujours des lieux d'éducation incitant à redécouvrir ces pratiques de l'informel propices aux apprentissages pour un devenir démocratique (Freire, 1998).

\section{CONCLUSION}

En conclusion, les obstacles récurrents rencontrés par les femmes immigrantes, notamment en ce qui concerne leur insertion socioprofessionnelle, soulèvent de nombreuses questions quant aux modalités d'accompagnement à privilégier. Face aux critiques qui dénoncent une tendance à considérer les personnes strictement sous l'angle des problèmes rencontrés et aux difficultés 
soulevées de relier les dimensions personnelles et structurelles, les résultats démontrent que les pratiques des intervenantes dans les organisations communautaires participantes permettent au contraire de prendre appui sur l'expertise expérientielle des femmes immigrantes.

Globalement, ces résultats démontrent que les pratiques s'ajustent aux femmes et favorisent le développement des savoirs. Par contre, il semble que les logiques de financement ne contribuent pas toujours à soutenir ces pratiques, ce qui a été surtout mentionné dans les organisations communautaires plus grandes ( $C$ et $D)$. Du côté des organisations communautaires situées en Belgique, les intervenantes ont d'ailleurs mentionné ressentir davantage les effets des politiques dites d'activation (Hamzaoui, 2013) qui entrent en contradiction avec certains principes à la base de leurs pratiques. Une piste de recherche serait justement de s'intéresser aux stratégies déployées par les intervenantes pour demeurer au plus près des fondements de leurs pratiques qui se rapprochent davantage d'un projet social à défendre. En témoignent les retombées identifiées dans le quartier où se situent ces organisations communautaires. Une autre piste consisterait à s'intéresser aux retombées identifiées par les femmes immigrantes de ces pratiques ${ }^{9}$.

CHAMBERLAND, MANON

Professeure

Département de travail social

Université du Québec en Outaouais

LE BOSSÉ, YANN

Professeur titulaire

Département des fondements et pratiques en éducation

Université Laval

\section{BIBLIOGRAPHIE}

Beaudoin, P. (2012). Portrait des femmes immigrées des minorités visibles recensées au Québec en 2006 et de leur participation au marché du travail, Québec, ministère de l'Immigration et des Communautés culturelles.

Belhassen-Maalaoui, A. (2008). L'impact de la non-reconnaissance des diplômes et des compétences: difficultés et impacts chez les femmes immigrantes, rapport de recherche, Montréal, Action-travail des femmes.

Bernier, C. (2011). Formation et employabilité. Regard critique sur l'évolution des politiques de formation de la main-d'œuvre au Québec, Québec, Presses de l'Université Laval.

\footnotetext{
${ }^{9}$ Pour prendre connaissance des retombées identifiées par les femmes immigrantes ayant fréquenté ces organisations, voir notamment :

Chamberland, M. et Le Bossé, Y. (sous presse). «Rendre visible l'invisible : des savoirs nouveaux et redécouverts par des femmes immigrantes au sein d'organisations communautaires », Alterstice - Revue internationale de la recherche interculturelle.
} 
Breton, M. (1999). « The Relevance of Structural Approach to Group Work with Immigrant and Refugee Women », Social Work With Groups, vol. 22, ns 2-3, p. 11-29.

Breton, M. (2004). « An Empowerment Perspective », dans C.D. Garvin, L.M. Guttiérrez, et M.J. Galinsky (dir.), Handbook of Social Work with Groups, New York, Guilford Press, p. 58-75.

Carr, E.S. (2003). « Rethinking Empowerment Theory Using a Feminist Lens: The Importance of Process », Affilia, vol. 18, $n^{\circ} 1$, p. 8-20.

Chicha, M.-T. (2009). Le mirage de l'égalité. Les immigrées hautement qualifiées à Montréal, rapport de recherche, Montréal, Immigration et Métropoles.

Cohen-Émérique (1993). « L'approche interculturelle dans le processus d'aide », Santé mentale au Québec, vol. 27, $\mathrm{n}^{\circ} 1$.

Conseil du statut de la femme (2005). Des nouvelles d'elles. Les femmes immigrées au Québec, Gouvernement du Québec.

Creswell, J.W. (2013). Qualitative Inquiry and Research Design, Los Angeles (CA), Sage publications.

Deville-Stoetzel, N., C. Montgomery et L. Rachédi (2012). «Quand la routine prend le dessus sur l'urgence : le rôle des réseaux dans les processus d'établissement en pays d'accueil », Alterstice - Revue internationale de la recherche interculturelle, vol. 2, nº 2, p. 79-90.

Duval, M., A. Fontaine, D. Fournier, S. Garon et J.-F. René (dir.) (2005). Les organismes communautaires au Québec. Pratiques et enjeux, Montréal, Gaëtan Morin Éditeur.

Fleury, D. (2007). Étude de la pauvreté et de la pauvreté au travail chez les immigrants récents au Canada, rapport final, Ottawa, Ressources humaines et développement social Canada.

Freire, P. (1998). Pedagogy of Freedom: Ethics, Democracy, and Civic Courage, Lanham (MD), Rowman \& Littlefield.

Gonin, A., J. Grenier et J.-A. Lapierre (2012). «Impasses éthiques des politiques sociales d'activation », Nouvelles pratiques sociales, vol. 25, $n^{0}$ 1, p. 166-186.

Gutiérrez, L.M. (1990). « Working with Women of Color: An Empowerment Perspective », Social Work, vol. 35, p. 149-153.

Gutiérrez, L.M. (1994). «Beyond Coping: An Empowerment Perspective on Stressful Life Events », Journal of Sociology and Social Welfare, vol. 21, nº 3, p. 201-219.

Hamzaoui, M. (2013). «La formation des adultes entre promotion sociale et logique d'adaptation », dans M. Hamzaoui, Les enjeux de la formation tout au long de la vie pour l'intervention sociale. Promotion sociale et citoyenne ou gestion de l'employabilité, Bruxelles, Éditions de l'IEIAS, p. 6-13.

Le Bossé, Y. (2004). «De "l'habilitation" au "pouvoir d'agir": vers une appréhension plus circonscrite de la notion d'empowerment », Nouvelles pratiques sociales, vol. $16, n^{0} 2, p .30-$ 51. 
Le Bossé, Y. (2010). Psychosociologie des sciences de l'orientation: Un point de vue interactionniste et stratégique, Québec, Éditions ARDIS.

Le Bossé, Y. (2012). Sortir de l'impuissance. Invitation à soutenir le développement du pouvoir d'agir des personnes et des collectivités, Québec, Éditions ARDIS.

Le Goff, F., C. McAll et C. Montgomery (2005). La transformation du communautaire. Expériences d'intervention auprès de jeunes sans emploi, Montréal, Éditions Saint-Martin.

Leclerc, C., B. Bourassa et O. Filteau. (2010). « Utilisation de la méthode des incidents critiques dans une perspective d'explicitation, d'analyse critique et de transformation des pratiques professionnelles », Éducation et francophonie, vol. 38, $\mathrm{n}^{\circ} 1, \mathrm{p} .11-31$.

Lee, J.A. (2001). The Empowerment Approach to Social Work Practice: Building the Beloved Community, New York (NY), Columbia University Press.

Legault, G., et G. Roy (2000). «Les difficultés des intervenants sociaux auprès des clientèles d'immigration récente », chap. 8, dans G. Legault, (dir.) L'intervention interculturelle, Boucherville, Gaëtan Morin Éditeur, p.185-202.

Lewis, E., L. Guttiérrez et I. Sakamoto (2001). «Women of Color », dans A. Gitterman (dir.) Handbook of Social Work Practice with Vulnerable and Resilient Populations, $2^{\mathrm{e}}$ éd., New York (NY), Columbia University Press, p. 820-839.

Lisboa, T.K. (2002). « Migrant Women in South Brazil and their Process of Empowerment », Migration, vol. 39-41, p. 113-135.

Luckerhoff, J., et F. Guillemette, (2012). « Conflits entre les exigences de la méthodologie de la théorisation enracinée (MTE) et les exigences institutionnelles en matière de recherche scientifique », dans J. Luckerhoff et F. Guillemette (dir.). Méthodologie de la théorisation enracinée. Fondements, procédures et usages, Québec, Presses de l'Université du Québec, p. 37-60.

Man, G. (2004). «Gender, Work and Migration: Deskilling Chinese Immigrant Women in Canada », Women's Studies International Forum, vol. 27, nº 2, p. 135-148.

McWhirter, E.H. (1994). Counseling for empowerment, Alexandria, VA: American Counseling Association.

McWhirter, E.H. (1998). « An Empowerment Model of Counsellor Education », Canadian Journal of Counseling/Revue canadienne de counseling, vol. 32, $\mathrm{n}^{\circ} 1, \mathrm{p} .12-16$.

Mongeau, J., G. Pinsonneault et D. Rose, (2007). Portrait économique des femmes immigrées recensées au Québec, Québec, ministère de l'Immigration et des Communautés culturelles.

Ninacs, W.A. (2008). Empowerment et intervention. Développement de la capacité d'agir et de la solidarité, Québec, Presses de l'Université Laval.

Normand, N., et D.-G. Tremblay (2005). Conciliation emploi-famille et intégration professionnelle. Le cas des femmes immigrées au Québec, note de recherche $n^{0} 2006-06$ de la Chaire de recherche du Canada sur les enjeux socio-organisationnels de l'économie du savoir, Montréal. 
OCDE (2011), Perspectives des migrations internationales 2011, Paris, Éditions OCDE. <doi : 10.1787/migr_outlook-2011-fr>, consulté le 13 janvier 2013.

Paugam, S. (2004). «Entretien du CRIEVAT-Laval avec Serge Paugam », dans G. Fournier et B. Bourassa (dir.), La précarité professionnelle. Effets individuels et sociaux, Saint-Nicolas (QC), Presses de l'Université Laval.

Pierre, M. (2005). « Les facteurs d'exclusion faisant obstacle à l'intégration socioéconomique de certains groupes de femmes immigrées au Québec », Nouvelles pratiques sociales, vol. 17, $\mathrm{n}^{\circ} 2$, p. 75-94.

Rappaport, J. (1987). « Terms of Empowerment/Exemplars of Prevention: Toward a Theory for Community Psychology », American Journal of Community Psychology, vol. 15, n 2, p. 121148.

René, J.-F. (2005). «Les organismes communautaires », dans M. Duval, A. Fontaine, D. Fournier, S. Garon et J.-F. René (dir.), Les organismes communautaires au Québec. Pratiques et enjeux, Montréal, Gaëtan Morin Éditeur.

René, J.-F. (2009). «L'individualisation de l'intervention : levier ou barrière à la prise en charge démocratique ? », Nouvelles pratiques sociales, vol. 22, $\mathrm{n}^{0}$ 1, p. 111-124.

Rifkin, S.B. (2003). « A Framework Linking Community Empowerment and Health Equity: It is a Matter of CHOICE », Journal of Health, Population and Nutrition, vol. 21, nº 3, p. 168-180.

Rifkin, S.B., F. Muller et W. Bichmann (1988). «Primary Health Care: On Measuring Participation », Social Science and Medicine, vol. 26, nº 9, p. 931-940.

Roy, S.N. (2003). « L'étude de cas », chap. 7, dans B. Gauthier (dir.), Recherche sociale. De la problématique à la collecte des données, Sainte-Foy, Presses de l'Université du Québec, p. 159-184.

Schellenberg, G., et H. Maheux (2007). Perspectives des immigrants sur leurs quatre premières années au Canada: Faits saillants des trois vagues de l'enquête longitudinale auprès des immigrants du Canada, «Tendances sociales canadiennes », n 11 008, Ottawa, Statistique Canada.

Schnapper, D. (2007). Qu'est-ce que l'intégration ?, Paris, Gallimard.

Tengland, P.-A. (2008). «Empowerment: A Conceptual Discussion », Health Care Analysis, vol. $16, n^{\circ} 2$, p. 77-96.

Tengland, P.-A. (2012). «Behavior Change or Empowerment: On the Ethics of HealthPromotion Strategies », Public Health Ethics, vol. 5, n² 2, p. 140-153.

Vatz-Laaroussi, M. (2008). Les familles immigrantes et leurs réseaux: des vecteurs de résilience intergénérationnels, Bulletin 46, Association pour la recherche interculturelle, p. 4354.

Vissandjée, B., et L. Maillet (2007). «L'empowerment et l'expérience de l'immigration au Canada. Multiples determinants dans une réalité complexe », dans H. Dorvil et R. Mayer, 
Problèmes sociaux, tome IV: Théories et méthodologies de l'intervention sociale, Québec, Presses de l'Université du Québec.

Wallerstein, N. (2006). What Is the Evidence of Effectiveness of Empowerment to Improve Health?, Copenhague, World Health Organization, WHO Regional Office for Europe.

Zentgraf, K.M. (2002). «Immigration and Women's Empowerment, Salvadorans in Los Angeles », Gender \& Society, vol. 16, $n^{\circ} 5$, p. 625-646. 